\title{
TAUBERIAN OPERATORS ON BANACH SPACES
}

\author{
NIGEL KALTON AND ALBERT WILANSKY
}

\begin{abstract}
A Tauberian operator: $E \rightarrow F$ (Banach spaces) is one which satisfies $T^{\prime \prime} g \in F, g \in E^{\prime \prime}$ imply $g \in E$. The action of such operators and their pre-images on compact sets is studied in order to compare "Tauberian" with "weakly compact", an opposite property. Properties related to range closed are introduced which force operators with Tauberian-like properties to be Tauberian. Classes of spaces appear for which Tauberian is equivalent to semi-Fredholm. One example of this is the historical reason for the definition of these operators.
\end{abstract}

1. Tauberian operators (\$2) appeared in response to a problem in summability (see [5]). Results on range closed Tauberian and co-Tauberian operators are given in [10]; our main results do not assume range closed.

We use standard Banach space notation: $B(E, F)$ is the set of bounded linear maps from $E$ to $F, E^{\prime}, \hat{E}$ are the dual of $E$ and the natural embedding of $E$ in $E^{\prime \prime}, T^{\prime}: F^{\prime} \rightarrow E^{\prime}$ is the adjoint of $T$, and $R T, N T$ are the range and nullspace of $T$. We write $E \subset E^{\prime \prime}$, identifying $E$ with $\hat{E}$ so that $T^{\prime \prime} \mid E=T$.

2. We call $T \in B(E, F)$ Tauberian if $T^{\prime \prime-1}[F] \subset E$, i.e. $g \in E^{\prime \prime}, T^{\prime \prime} g \in F$ imply $g \in E$. It is immediate that a Tauberian operator has the property

$$
\text { (N): } g \in E^{\prime \prime}, T^{\prime \prime} g=0 \text { imply } g \in E \text {. }
$$

It is known that $(\mathrm{N})$ implies

$$
\text { (R): } N T \text { is reflexive. }
$$

For range closed operators the three conditions are equivalent [5], [10]. Parts of this equivalence hold more generally as follows: (We omit the proofs.) (i) The following three conditions are equivalent. (a) $T$ is Tauberian, (b) $T$ has property (N) and $T[D]$ is closed ( $D$ is the unit disc), (c) $T$ has property $(\mathrm{N})$ and the closure of $T[D]$ is included in the range of $T$. (ii) $T$ has property $(\mathrm{N})$ if and only if it has property (R) and the range of $T^{\prime}$ has norm closure equal to its $w^{*}$ closure.

For $T$ one-to-one these results apply to the map given in [3, II, Lemma 1(iii)]. It also follows that any adjoint map with property (N) must be Tauberian. By considering the quotient map (which is automatically Tauberian) we see that if $T \in B(E, F)$ is Tauberian and $S \subset F$ is reflexive, then $T^{-1}[S]$ is reflexive. This generalizes (R).

Received by the editors July 7, 1975 and, in revised form, September 29, 1975.

AMS (MOS) subject classifications (1970). Primary 47B05, 47B30.

Key words and phrases. Tauberian, operators, Banach space.

C American Mathematical Society 1976 
3. Tauberian operators are, in a sense, opposite to weakly compact operators since $T \in B(E, F)$ is weakly compact if and only if $R T^{\prime \prime} \subset F$. Thus the set of Tauberian operators lies in the complement of a closed subspace of $B(E, F)$, a closed ideal if $F=E$. Other nonweakly compact operators can be obtained by taking adjoints of Tauberian operators. There are examples of pairs of Banach spaces, $E, F$, neither one reflexive, such that no operator in $B(E, F)$ is Tauberian). It suffices to consider a pair such that every operator is weakly compact.

We now characterize Tauberian operators (internally) in terms of their mapping of compact sets. This may be compared with the fact that $T$ is weakly compact if and only if it maps all bounded sets into relatively weakly compact sets. We begin with a criterion for property $(\mathrm{N})$.

3.1. TheOREM. Let $E, F$ be Banach spaces, $T \in B(E, F)$. Then $T$ has property (N) if and only if every bounded sequence $\left\{x_{n}\right\}$ in $E$ with $T x_{n} \rightarrow 0$ in $F$ has a weakly convergent subsequence.

Necessity. For any $w^{*}$ cluster point $z$ of $\left\{x_{n}\right\}, T^{\prime \prime} z=0$ since $T^{\prime \prime} x_{n}=T x_{n}$ $\rightarrow 0$ in norm. Hence $z \in E$ and it follows that $\left\{x_{n}\right\}$ is relatively weakly compact, hence relatively weakly sequentially compact, by the EberleinSmulian theorem.

Sufficiency. Suppose $g \in E^{\prime \prime}, T^{\prime \prime} g=0$. We may assume $\|g\|=1$. There exists a net $x$ in $D$, the unit disc of $E$, with $x \rightarrow g, w^{*}$. Then $T x=T^{\prime \prime} x$ $\rightarrow T^{\prime \prime} g=0, w^{*}$ and so $T x \rightarrow 0$, weakly. Thus if $C$ is any convex subset of $E$ such that $x \in C$ eventually, it follows that 0 is in the norm closure of $T C$. Writing $x=\left\{x^{\alpha}: \alpha \in A\right\}$; for each $\alpha \in A$, let $C_{\alpha}$ be the convex hull of $\left\{x^{\gamma}: \gamma \geqslant \alpha\right\}$. As just mentioned, each $C_{\alpha}$ contains a sequence $\left\{c_{\alpha}^{n}\right\}$ with $\left\|T c_{\alpha}^{n}\right\| \rightarrow 0$. By hypothesis $\left\{c_{\alpha}^{n}\right\}$ has a subsequence converging weakly to $c_{\alpha}$, say. Clearly $T c_{\alpha}=0$. Now $\left\{c_{\alpha}: \alpha \in A\right\}$ is relatively weakly sequentially compact [each sequence $\left\{v_{n}\right\}$ in it has $T v_{n}=0$ ], hence, by Eberlein-Smulian, relatively weakly compact. Thus $c_{\alpha}$ has a weak cluster point $c$. The proof is concluded by showing $g=c$. This is clear since $c_{\alpha} \rightarrow g, w^{*}$, and $c$ is a $w^{*}$ cluster point of $c_{\alpha}$.

3.2. Theorem. Let $E, F$ be Banach spaces, $T \in B(E, F)$. The following are equivalent:

(i) $T$ is Tauberian.

(ii) For every bounded set $B \subset E$ such that $T B$ is relatively weakly compact, $B$ is relatively weakly compact.

(iii) For every bounded set $B \subset E$ such that $T B$ is relatively compact, $B$ is relatively weakly compact.

(i) implies (ii). Let $T$ be Tauberian, $B$ bounded $\subset E, T B$ relatively weakly compact. Let $x$ be a net in $B$. Then $x$, being a bounded net in $E^{\prime \prime}$ has a $w^{*}$ convergent subnet which we may assume to be $x$ itself; say $x \rightarrow g \in E^{\prime \prime}, w^{*}$. Also $x$ has a subnet, which we may again assume to be $x$ itself, such that $T x \rightarrow y \in F$ weakly. Then $T^{\prime \prime} g=w^{*} \lim T^{\prime \prime} x=w^{*} \lim T x=y$. Since $T$ is Tauberian, $g \in E$. Then $x \rightarrow g$ weakly. Thus $B$ is relatively weakly compact.

(ii) implies (iii). If $T B$ is relatively norm compact its norm closure is a 
compact, hence weakly compact set which includes $T B$; hence $T B$ is relatively weakly compact.

(iii) implies (i). Let $D$ be the unit disc of $E$ and $y \in \overline{T D}$. Choose $\left\{x_{n}\right\}$ in $D$ with $T x_{n} \rightarrow y$. By hypothesis $\left\{x_{n}\right\}$ has a weak cluster point $x$; clearly $x \in D$ and $y=T x$, so $y \in R T$. By $\S 2$, (i), it remains to prove that $T$ has property $(\mathrm{N})$. This follows from 3.1 and the Eberlein-Smulian theorem.

4. We call $T \in B(E, F)$ a semi-Fredholm operator (as in [8]) and write $T \in \Phi_{+}$if $T$ is range closed and $\operatorname{dim} N T<\infty$. Such operators are Tauberian and in the eponymic case the converse is true, see 4.3 and [5]. Such operators are also discussed in [4], [9]. For the next result, due to Yood, Wolf, Basley, Schubert et al., see $[6,4.11,4.12]$.

4.1. THEOREM. The following are equivalent for $T \in B(E, F)$ :

(i) $T \in \Phi_{+}$.

(ii) For every bounded set $B \subset E$ such that $T B$ is compact, $B$ is relatively compact.

(iii) Every bounded sequence $\left\{x_{n}\right\}$ in $E$ with $T x_{n} \rightarrow 0$ has a convergent subsequence.

4.2. Theorem. Let $T \in B(E, F)$ be Tauberian. Then $T \in \Phi_{+}$if and only if $T \mid R \in \Phi_{+}$for all reflexive subspaces $R$ of $E$.

That $T \mid R \in \Phi_{+}$for any closed $R$ is by 4.1 . Conversely suppose that $T \notin \Phi_{+}$. The hypothesis implies that $\operatorname{dim} N T<\infty$ so we may restrict $T$ to the complement of $N T$, i.e. we may assume $T$ is one-to-one. By 4.1 we can find $\left\{x_{n}\right\},\left\|x_{n}\right\|=1, T x_{n} \rightarrow 0$. By [2, p. 156], $\left\{x_{n}\right\}$ has a basic subsequence, which we may assume to be $\left\{x_{n}\right\}$ itself, with $\left\|T x_{n}\right\| \leqslant 2^{-n}$. Let $X$ be the linear closure of $\left\{x_{n}\right\}$. Then $T \mid X$ is compact and Tauberian. Now let $D$ be the unit disc of $X$. By $3.2, D$ is relatively weakly compact hence $X$ is reflexive. But $T \mid X$ is not range closed.

4.3. Corollary. Suppose $E$ has no reflexive infinite dimensional subspace; then for a map $T \in B(E, F), T$ is Tauberian if and only if $T \in \Phi_{+}$.

5. The result obtained by taking $E=c$ in 4.3 is an extension of the BergCrawford-Whitley theorem. (See [5].) We may also take $E=l$ (space of absolutely convergent series) in 4.3 but Theorem 5.1 is better. See also 5.2.

5.1. TheOREM. For any Banach space $F$ and $T \in B(l, F)$ the following are equivalent.

(i) $T$ is Tauberian.

(ii) $T$ has property $(\mathrm{N})$.

(iii) $T \in \Phi_{+}$.

By 4.3, (i) and (iii) are equivalent. If (ii) holds, $T$ has property (R) and so its null-space is finite dimensional. Since the restriction of $T$ to a closed subspace satisfies (N), we may assume that $T$ is one-to-one; our assumption is then that $T^{\prime \prime}$ is also one-to-one and so $T^{\prime}: F^{\prime} \rightarrow l^{\infty}$ has dense range. By a remark of Beurling (see [1, Theorem 3]), $T^{\prime}$ is onto; hence $T$ is an isomorphism. (The equivalence of (i), (ii) also follows from 3.1, 3.2.) 
The next result (which is false for $E=c$ ) generalizes the equivalence of (i) and (ii) in 5.1. The referee has pointed out that this proof may be simplified by citing [7].

5.2. THEOREM. Let $E$ be a weakly sequentially complete Banach space, $F$ any Banach space, and $T \in B(E, F)$. Then $T$ is Tauberian if and only if it has property $(\mathrm{N})$.

Applying Eberlein-Smulian to 3.2(iii), it is sufficient to show that if $\left\{x_{n}\right\}$ in $E$ is bounded and $\left\{T x_{n}\right\}$ is convergent, then $\left\{x_{n}\right\}$ has a weakly convergent subsequence. As in 5.1, we may restrict ourselves to the linear closure of $\left\{x_{n}\right\}$, thus we may assume that $E$ is separable. Let $R=N T$; it is reflexive since $T$ has property (N). Let $q: E \rightarrow E / R$ be the quotient map. We first show that $\left\{q\left(x_{n}\right)\right\}$ is weakly Cauchy. [If this is false, there exist increasing sequences $\{m(k), n(k)\}$ and $f \in(E / R)^{\prime}$ with $\left|f\left[q\left(x_{m(k)}\right)-q\left(x_{n(k)}\right)\right]\right| \geqslant 1$. Let $w$ be a weak cluster point of $\left\{x_{m(k)}-x_{n(k)}\right\}$ by 3.1. Then $T w=0$, so $w \in R$. Thus $q(w)=0$ which contradicts $|f[q(w)]| \geqslant 1$.] This means that $\left\{f\left(x_{n}\right)\right\}$ is convergent for all $f \in R^{\perp} \subset E^{\prime}$. Now $R^{\prime}=E^{\prime} / R^{\perp}$ is separable since $R^{\prime \prime}=R$ is, so there exists a sequence $\left\{f_{n}\right\} \subset E^{\prime}$ such that the linear closure of $R^{\perp}$ $\cup\left\{f_{1}, f_{2}, \ldots\right\}$ is $E^{\prime}$. Select a subsequence $\left\{u_{n}\right\}$ of $\left\{x_{n}\right\}$ such that $\lim _{n} f_{i}\left(u_{n}\right)$ exists for each $i$. [This is possible since $\left\{x_{n}\right\}$ is bounded.] Since also $\left\{f\left(u_{n}\right)\right\}$ is convergent for all $f \in R^{\perp}$ and $\left\{u_{n}\right\}$ is bounded, it follows that $\left\{u_{n}\right\}$ is weakly Cauchy, hence weakly convergent.

6. We ask the following questions.

6.1. When is it true that $T$ is Tauberian if and only if $T^{\prime \prime}$ is? (For example this is true if $T$ is range closed.)

6.2. Which Banach spaces $E$, other than those mentioned in 4.3 have the property that a Tauberian map must be range closed? (Clearly every Tauberian map on $E$ has finite dimensional null-space if and only if $E$ has no infinite dimensional reflexive subspace.)

6.3. When is the induced map $T: E^{\prime \prime} / E \rightarrow F^{\prime \prime} / F$ an isomorphism? (It is oneto-one if and only if $T$ is Tauberian. For range closed maps see [10].)

6.4. We feel that a Tauberian map $T: C(X) \rightarrow C(Y)$ must be close to an isomorphism in some sense.

\section{REFERENCES}

1. W. G. Bade and P. C. Curtis, Jr., Embedding theorems for commutative Banach algebras, Pacific J. Math. 18 (1966), 391-409. MR 34 \# 1878.

2. C. Bessaga and A. Pełczyński, On bases and unconditional convergence of series, Studia Math. 17 (1958), 151-164. MR 22 \#5872.

3. W. J. Davis, T. Figiel, W. B. Johnson and P. Pełczyński, Factoring weakly compact operators, J. Functional Analysis 17 (1974), 311-327.

4. R. de Vos, $\theta$ maps between FK spaces, Math Z. 129 (1975), 287-298.

5. D. J. H. Garling and A. Wilansky, On a summability theorem of Berg, Crawford and Whitley, Proc. Cambridge Philos. Soc. 71 (1972), 495-497. MR 45 \#4014.

6. A. Lebow and M. Schechter, Semigroups of operators and measures of noncompactness, J. Functional Analysis 7 (1971), 1-26. MR 42 \#8301.

7. H. P. Rosenthal, $A$ characterization of Banach spaces containing $l^{1}$, Proc. Nat. Acad. Sci. U.S.A. 71 (1974), 2411-2413.

8. M. Schechter, Principles of functional analysis, Academic Press, New York, 1971. 
9. A. Wilansky, Semi-Fredholm maps of FK spaces, Math. Z. 144 (1975), 9-12.

10. K.-W. Yang, The generalized Fredholm operators, Trans. Amer. Math. Soc. 216 (1976), 313-326.

Department of Mathematics, University College of Swansea, Swansea, Wales, Great BRITAIN

Department of Mathematics, Lehigh University, Bethlehem, Pennsylvania 18015 